\title{
Methyl-DNA immunoprecipitation (MeDIP): Hunting down the DNA methylome
}

\author{
Filipe V. Jacinto, Esteban Ballestar, and Manel Esteller \\ Cancer Epigenetics Group, Spanish National Cancer Research Centre (CNIO), Madrid, Spain
}

One of the most challenging projects in the field of epigenetics is the generation of detailed functional maps of DNA methylation in different cell and tissue types in normal and disease-associated conditions. This information will help us not only understand the role of DNA methylation but also identify targets for therapeutic treatment. The completion of the various epigenome projects depends on the design of novel strategies to survey and generate detailed cartograms of the DNA methylome. Methyl-DNA immunoprecipitation (MeDIP) assays, in combination with hybridization on high-resolution microarrays or high-throughput sequencing (HTS) techniques, are excellent methods for identifying methylated CpG-rich sequences. We provide a critical overview of different genome-wide techniques for DNA methylation analysis and propose that MeDIP assays may constitute a key method for elucidating the hypermethylome of cancer cells.

\section{Introduction}

DNA methylation is a major epigenetic modification with direct implications for the establishment of expression patterns in multicellular organisms (1). In mammals, it is restricted to the genomic context of $\mathrm{CpG}$ dinucleotides, which cluster in repetitive sequences and in regions known as CpG islands, most of which overlap, or are near, the transcription start site of genes (2). Nevertheless, under normal conditions, most promoter $\mathrm{CpG}$ islands remain unmethylated (3). Methylation and subsequent repression of expression are confined to a relatively small set of genes, including those in the inactive X-chromosome of females (4), imprinted genes (5), and tissue-specific genes $(6,7)$. Gene silencing by the methylation of promoter $\mathrm{CpG}$ islands involves the modification of histones not only through the direct recruitment of histone-modifying activities by DNA methyltransferases (8), but also through other nuclear factors such as methyl-CpG binding domain (MBD) proteins $(9,10)$. It is of particular note that the role of DNA methylation in the proper functioning of cells was recognized in the course of intensive studies in different disease contexts. Thus, the recognition that cancer cells undergo dramatic changes in the levels and distribution of DNA methylation has been largely responsible for prompting the massive research effort into unveiling the identity of the genes that undergo this type of epigenetic deregulation as well as the functional consequences and mechanisms involved. Currently, the DNA methylationdependent silencing of many tumor-suppressor genes in cancer is recognized as being a major mechanism of gene inactivation that complements genetic lesions (11). An interesting example that has stimulated great interest in the essential role of the proper establishment of DNA methylation patterns during development comes from the recognition of the functional consequences of the genetic loss of DNA methyltransferase $3 \mathrm{~b}$ in immunodeficiencycentromere instability-facial anomalies syndrome (ICF). Known epigenetic alterations in this syndrome include the loss of methylation in a number of repetitive sequences (12). Other disorders, such as the imprinting disorders Prader-Willi or Angelman syndromes, or the alpha-thalassemia/mental retardation, X-linked (ATRX) syndrome, are accompanied by changes in DNA methylation (13). Despite the recognition of a fundamental role for DNA methylation patterns in the correct establishment of gene expression patterns, relatively little information is available about the physiological role of promoter methylation of genes, and very few genes have been confirmed as being responsible for tissue-specific $(6,7,14)$ or developmentally associated (15) DNA methylation. The availability of techniques to map methylation profiles of genes in normal and diseaseassociated cells will surely contribute to a better understanding of the implications of methylation changes in the regulation of gene expression and nuclear function.

\section{Developing Strategies to Characterize DNA Methylation Profiles}

Cancer epigenetics research has had an enormous effect on the development of strategies for identifying methylated sequences. For years, classical tumor-suppressor genes for which genetic lesions had been described, such as VHL, p73, or p16(INK4a), constituted the only source of candidate genes used for performing analysis on the methylation status of their promoter CpG islands (16). The systematic use of this strategy led to the identification of a number of genes that are epigenetically inactivated in cancer and to the notion that specific DNA hypermethylation patterns occur in cancer in a tumor-specific fashion (16). These 


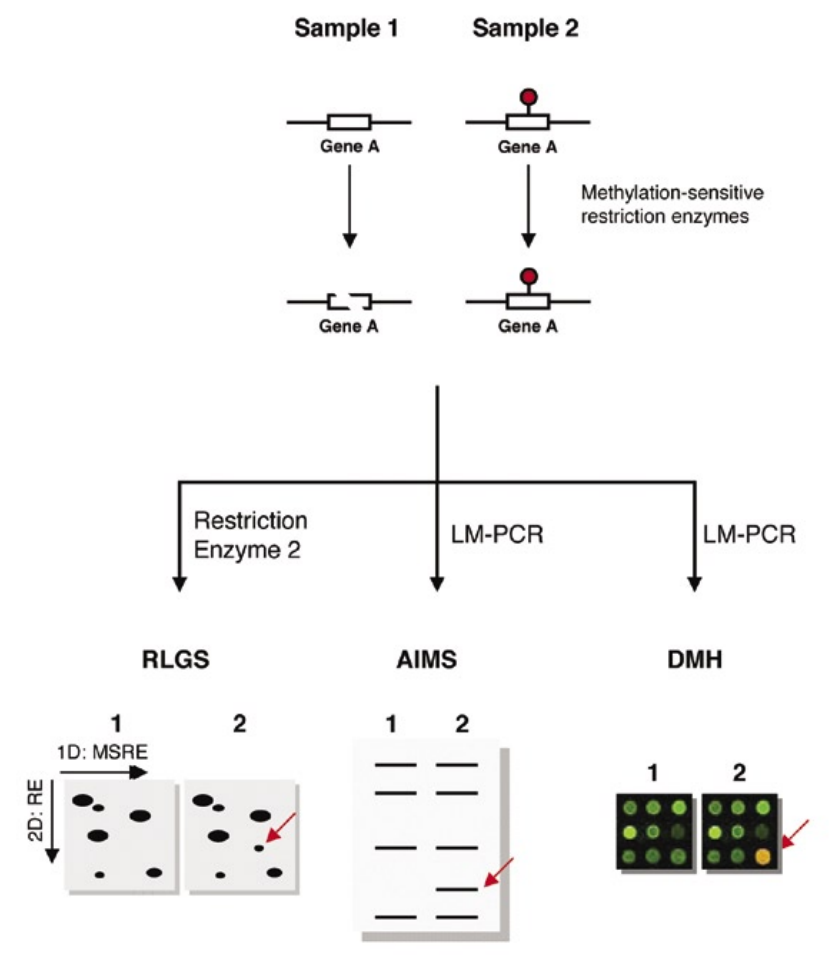

Figure 1. Scheme depicting three common methods for methylation profiling based on the use of methylation-sensitive restriction enzymes. A target site for methylation-sensitive restriction enzymes (MSRE) is shown (empty box) with two alternative methylation statuses (DNA methylation is represented by a red circle) of two possible samples (cancer versus normal tissue, tissue 1 and tissue 2, etc). In restriction landmark genomic scanning (RLGS) (bottom left panel), a second digestion is performed and the DNA fragments are analyzed by two-dimensional (2-D) gel electrophoresis. In amplification of intermethylated sites (AIMS) (bottom center), MSRE digestion is followed by ligation-mediated PCR and separation on a high-resolution denaturing gel. In differential methylation hybridization (DMH) (bottom right panel), amplified products of the two samples are competitively hybridized on a genomic microarray.

candidate gene-based approaches also highlighted the need to develop genomic strategies to obtain comprehensive DNA methylation profiles of cancer cells. Research efforts in this direction have yielded several methods.

The first group of approaches was based on the digestion of genomic DNA with restriction enzymes unable to digest the methylated version of their CpG-containing target sites. These strategies allow researchers to make a direct comparison of differentially methylated sites between two/various cell types (Figure 1). In combination with digestion with methylation-sensitive restriction enzymes, different methods are used to resolve or analyze the resulting fragments. For instance, in restriction landmark genomic scanning (RLGS) $(17,18)$, fragments resulting from digestion are separated in a two-dimensional (2-D) gel electrophoresis (Figure 1). When comparing two different cell types, differential spots need to be individually analyzed for their methylation status. Other methods use a combination of methylation-sensitive restriction enzymes with ligation- mediated PCR amplification. An excellent example is that of the amplification of intermethylated sites (AIMS) (19).

More recent strategies consist in the generation of DNA fragments through digestion of genomic DNA with methylation-sensitive restriction enzymes combined with hybridization on genomic microarrays (20) (Figure 1). Again, this method is useful to perform a direct comparison between two different cell types for which differential methylation throughout the genome is expected.

All these methods have been shown to be useful for methylome profiling $(18,19,20)$. However, the need of DNA sequencing in the case of both RLGS and AIMS to determine the sequence identity of differential spots or bands (Figure 1) has been a practical limitation of these two methods. The recent availability of high-throughput sequencing (HTS) techniques may overcome this constraint.

\section{Using Antibodies to Isolate Methylated DNA: Methyl-DNA Immunoprecipitation}

An additional limitation of restriction enzyme-based approaches is that only particular sequence motifs can be analyzed because specific restriction sites are required to be present. In order to circumvent this constraint, and considering that the major goal is the isolation/fractionation of methylated DNA, several strategies involving the specific interaction of proteins with methylated DNA have been designed. One attractive target for this type of approach is the MBD family of proteins, which are chosen for their selectivity in binding methylated DNA. Strategies exploiting this group of proteins include the use of an MBD domain fused to a human IgG (21), or the use of affinity columns where MBD proteins are bound to a Sepharose matrix (22). Alternatively, chromatin immunoprecipitation with anti-MBD antibodies coupled to hybridization in genomic microarrays has also proved to be useful for isolating methylated DNA sequences (23). This method also provides information about the specific nuclear factors implicated and the mechanism that leads to gene silencing in hypermethylated genes.

More recently, Weber et al. developed an immunocapturing approach based on the direct immunoprecipitation of methylated DNA (24) (Figure 2). In this assay, named methyl-DNA immunoprecipitation (MeDIP), a monoclonal antibody raised against 5-methylcytidine $(5 \mathrm{mC})$ is used to purify methylated DNA. The assay is very simple. In brief, genomic DNA purified by standard procedures is sheared through sonication to produce random fragments. Sonication conditions must be optimized to yield fragments ranging in size between 300 and $600 \mathrm{bp}$. The generation of small fragments is key to guaranteeing efficient immunoprecipitation and a reasonable level of resolution that is necessary for further characterization. After fragmentation, DNA must be denatured at $95^{\circ} \mathrm{C}$ in order to yield single- 
stranded DNA fragments, as the anti- $5 \mathrm{mC}$ has a higher affinity for this form of the $5 \mathrm{mC}$-containing DNA. The rest of the assay is very similar to a classical immunoprecipitation protocol and involves the use of protein G coupled to standard or magnetic beads as well as multiple washes following incubation with the anti-5mC antibody. Immunoprecipitated DNA can then be used for individual analysis of the methylation status of a particular gene by employing specific primers targeting the specified gene, although the importance of this technique arises from the possibility of using immunoprecipitated DNA to hybridize high-resolution genomic microarrays.

The study of Weber et al. (24), in which they used MeDIP for the first time, revealed the usefulness of this technique for obtaining high-resolution maps of the human methylome. For instance, it confirmed that the inactive X-chromosome in females is hypermethylated at $\mathrm{CpG}$ islands at a chromosome-wide level (24). This study also showed, for instance, that the global distribution of $\mathrm{CpG}$ methylation in the colon cancer model used is markedly similar to that of primary fibroblasts. It also confirmed that DNA methylation changes in $\mathrm{CpG}$ islands of cancer cells primarily involves hypermethylation events and that hypomethylation is a rare event. A useful application of MeDIP combined to hybridization with microarrays is the possibility of performing parallel chromatin immunoprecipitation experiments using the same platform (25) to investigate correlation between DNA methylation patterns and histone modification marks.

\section{Using MeDIP for Mapping the Cancer DNA Hypermethylome}

As mentioned above, epigenetic changes in cancer have been at the forefront of epigenetics research for over a decade. Besides the relevance of identifying targets of epigenetic inactivation at a genome-wide level in cancer, all tumor types exhibit profoundly disrupted DNA methylation profiles. MeDIP in combination with microarray technology or other novel high-throughput strategies suits the need for high-resolution analysis of the hypermethylome.

For instance, colon carcinoma and prostate cancer cells have recently been analyzed using MeDIP combined with a microarray containing over 13,000 promoters (26). This strategy allowed the identification of a large number of genes with hypermethylated $\mathrm{CpG}$ islands. The bioinformatic analysis of these genes allowed the determination of general features regarding gene category or nucleotide sequence and motifs that allowed the authors to propose the existence of an instructive mechanism for de novo DNA methylation (26).

We have recently applied the MeDIP approach to investigate the profile of promoter methylation in colon cancer cells (27). The parallel analysis of the same colorectal cancer cell line, in which the two major DNA methyltransfer-

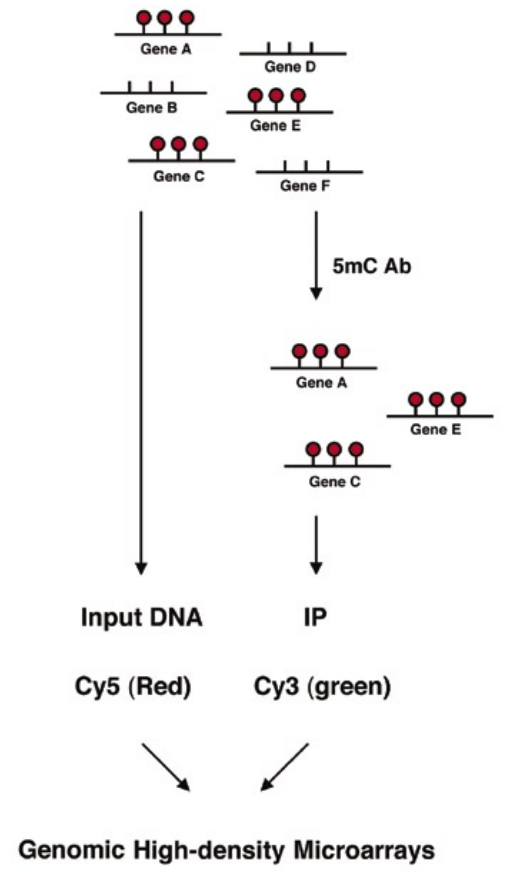

Figure 2. Methyl-DNA immunoprecipitation (MeDIP) assay. Genomic DNA (Input) sheared through sonication is immunoprecipitated with antibody against 5-methylcytidine. Methylated $\mathrm{CpGs}$ are represented by red circles. The methylated DNA is then labeled with Cy3 (green) and used for cohybridization with input DNA (labeled with Cy5, red) on a microarray.

ases, DNMT1 and DNMT3b, have been genetically disrupted allows the unambiguous identification of $\mathrm{CpG}$ islands that lose hypermethylation when DNMT1 and DNMT3b are removed (Figure 3). Interestingly, our experimental approach showed that only a limited number of genes (126 genes) are enriched when immunoprecipitating this colon cancer cell line with anti-5mC. The order of magnitude of identified targets (27), similar to that obtained by Weber et al. (24), indicates that there may be fewer hypermethylated promoter $\mathrm{CpG}$ islands in a particular tumor type than previously hypothesized.

Individual analysis by bisulfite sequencing with specific primers leads to two conclusions: first, a significant proportion of the immunoprecipitated genes can be validated using standard bisulfite genomic sequencing; second, the strategy is appropriate for the parallel comparison of two/multiple cell lines with differential expression of an epigenetic enzyme (Figure 3).

One caveat with affinity approaches is that methylated CpG-rich sequences may give higher enrichments than methylated CpG-poor sequences. In general, aberrant hypermethylation of genes in cancer cells occurs throughout their entire $\mathrm{CpG}$ island at the promoter. Since $\mathrm{CpG}$ islands are regions of high CpG density, cancer cells and genes that suffer promoter $\mathrm{CpG}$ island hypermethylation represent an excellent system for this type of analysis. In contrast, promoters with a low $\mathrm{CpG}$ dinucleotide content 


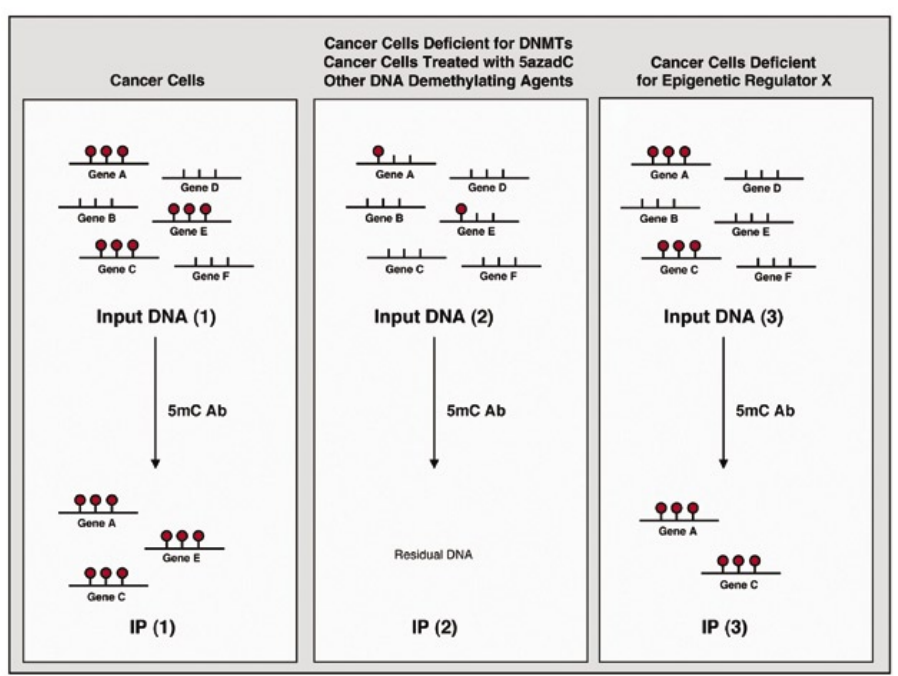

Figure 3. Different strategies for which MeDIP can be used in order to investigate DNA methylation changes in cancer cells. Methylated CpGs are represented by red circles. In all panels the input fraction is represented on the top part and the immunoprecipitated (IP) fraction on the bottom part. After generation of the input and IP fractions, hybridization on genomic microarrays or high-throughput sequencing can be performed. (A) Immunoprecipitation with antibody against 5-methylcytidine $(5 \mathrm{mC}$ $A b)$ results in generation of a fraction enriched in methylated sequences [IP (1)]. (B) The assays can be applied to cells treated that are deficient in DNA methyltransferases (29), or treated with a known (5-Aza-Deoxycytidine, 5azadC) or novel DNA demethylating compound. (C) MeDIP is also useful to test genes for which a potential role in establishment of DNA methylation patterns is suspected.

and subtle methylation changes do not seem to be a good target for such studies.

Several advantages make MeDIP a useful technique for profiling the hypermethylome of cancer cells. Firstly, since genomic DNA is the necessary source material, it is possible to used stored specimens, including DNA from frozen or paraffin-embedded tumor samples. In fact, MeDIP can be used to detect premalignant epigenetic lesions at the genomic level (27), making this a powerful diagnostic technique. One of the potential limitations, which perhaps applies to genomic microarrays, can be circumvented by combining MeDIP with the HTS technologies that are now available. This type of strategy is similar to that of combining chromatin immunoprecipitation assays with HTS to obtain high-resolution maps of histone modifications (28).

A novel high-throughput array-based method for analyzing the methylation status of hundreds of preselected genes has recently been developed (29). At the current level of development, this type of array enables the analysis of several thousand genes. The results obtained with this method demonstrate its effectiveness for reliably profiling many $\mathrm{CpG}$ sites in parallel, by which informative methylation markers may be identified. As before, the major limitation of this technique is the platform on which targets are selected. In this sense, the possibility of combining MeDIP with HTS makes this the preferable strategy for advancing the genome-wide analysis of the DNA methylome.

\section{Acknowledgments}

M.E. is funded by the Education and Science Department of the Spanish Government (grant no. I+D+I MCYT08-03 and Consolider grant no. MEC09-05) and by the European Union (grant no. CANCERDIP 200620 FP7). E.B. is funded by Ministerio de Educacion y Ciencia (MEC) (grant no. BFU2004-02073/BMC). F.V.J. is a recipient of a GABBA Fellowship from the Foundation for Science and Technology, Portugal.

\section{References}

1.Jaenisch, R., and A. Bird. 2003. Epigenetic regulation of gene expression: how the genome integrates intrinsic and environmental signals. Nature Genet. 33:Suppl:245-254.

2.Gardiner-Garden, M. and M. Frommer. 1987. CpG islands in vertebrate genomes. J. Mol. Biol. 196: 261-282.

3.Siegfried, Z. and H. Cedar. 1997. DNA methylation: a molecular lock. Curr. Biol. 7:R305-307.

4.Wolf, S.F. and B.R. Migeon. 1982. Studies of X chromosome DNA methylation in normal human cells. Nature 295: 667671.

5.Reik, W., A. Collick, M.L. Norris, S.C. Barton, and M.A. Surani. 1987. Genomic imprinting determines methylation of parental alleles in transgenic mice. Nature 328: 248-251.

6.Futscher, B.W., M.M. Oshiro, R.J. Wozniak, N. Holtan, C.L. Hanigan, H. Duan, and F.E. Domann 2002. Role for DNA methylation in the control of cell type specific maspin expression. Nature Genet. 31:175-179.

7.Song, F., J.F. Smith, M.T. Kimura, A.D. Morrow, T. Matsuyama, H. Nagase, and W.A. Held. 2005. Association of tissue-specific differentially methylated regions (TDMs) with differential gene expression. Proc. Natl. Acad. Sci. USA 102:3336-3341.

8.Burgers, W.A., F. Fuks, and T. Kouzarides. 2002. DNA methyltransferases get connected to chromatin. Trends Genet. 18:275-277.

9. Hendrich, B. and A. Bird. 1998. Identification and characterization of a family of mammalian methyl-CpG binding proteins. Mol. Cell. Biol. 18:6538-6547.

10. Ballestar, E. and M. Esteller. 2005. Methyl-CpG-binding proteins in cancer: blaming the DNA methylation messenger. Biochem. Cell Biol. 83:374-384.

11. Esteller, M. 2007. Epigenetic gene silencing in cancer: the DNA hypermethylome. Hum. Mol. Genet. 16:R50-59.

12. Kondo, T., M.P. Bobek, R. Kuick, B. Lamb, X. Zhu, A. Narayan, D. Bourc'his, E. Viegas-Péquignot, M. Ehrlich, and S.M. Hanash. 2000. Whole-genome methylation scan in ICF syndrome: hypomethylation of non-satellite DNA repeats D4Z4 and NBL2. Hum. Mol. Genet. 9:597-604.

13. Egger, G., G. Liang, A. Aparicio, and P.A. Jones. 2004. Epigenetics in human disease and prospects for epigenetic therapy. Nature 429:457-463.

14. Rakyan, V.K., T. Hildmann, K.L. Novik, J. Lewin, J. Tost, A.V. Cox, T.D. Andrews, K.L. Howe, T. Otto, et al. 2004. DNA methylation profiling of the human major histocompatibility complex: a pilot study for the human epigenome project. PLoS Biol. 2:e405. 
15. Eckhardt, F., J. Lewin, R. Cortese, V.K. Rakyan, J. Attwood, M. Burger, J. Burton, T.V. Cox, R. Davies, T.A. Down, et al. 2006. DNA methylation profiling of human chromosomes 6, 20 and 22. Nature Genet. 38:1378-1385.

16. Esteller, M., P.G. Corn, S.B. Baylin, and J.G. Herman. 2001. A gene hypermethylation profile of human cancer. Cancer Res. 61:3225-3229.

17. Plass, C., H. Shibata, I. Kalcheva, L. Mullins, N. Kotelevtseva, J. Mullins, R. Kato, H. Sasaki, S. Hirotsune, Y. Okazaki, W.A. Held, Y. Hayashizaki, and V.M. Chapman. 1996. Identification of Grf1 on mouse chromosome 9 as an imprinted gene by RLGS-M. Nature Genet. 14:106-109.

18.Costello, J.F., M.C. Frühwald, D.J. Smiraglia, L.J. Rush, G.P. Robertson, X. Gao, F.A. Wright, J.D. Feramisco, et al. 2000. Aberrant $\mathrm{CpG}$-island methylation has non-random and tumourtype-specific patterns. Nature Genet. 24:132-138.

19. Frigola, J., M. Ribas, R.A. Risques, and M.A. Peinado. 2002. Methylome profiling of cancer cells by amplification of intermethylated sites (AIMS). Nucleic Acids Res 30:e28.

20.Huang, T. H., M.R. Perry, and D.E. Laux. 1999. Methylation profiling of $\mathrm{CpG}$ islands in human breast cancer cells. Hum. Mol. Genet. 8:459-470.

21. Gebhard, C., L. Schwarzfischer, T.H. Pham, E. Schilling, M. Klug, R. Andreesen, and M. Rehli. 2006. Genome-wide profiling of $\mathrm{CpG}$ methylation identifies novel targets of aberrant hypermethylation in myeloid leukemia. Cancer Res 66:6118-6128.

22.Selker, E.U., N.A. Tountas, S.H. Cross, B.S. Margolin, J.G. Murphy, A.P. Bird, and M. Freitag. 2003. The methylated component of the Neurospora crassa genome. Nature 422:893-897.

23.Ballestar, E., M.F. Paz, L. Valle, S. Wei, M.F. Fraga, J. Espada, J.C. Cigudosa, T. H.-M. Huang, and M. Esteller. 2003. Methyl$\mathrm{CpG}$ binding proteins identify novel sites of epigenetic inactivation in human cancer. EMBO J. 22:6335-6345.

24.Weber, M., J.J. Davies, D. Wittig, E.J. Oakeley, M. Haase, W.L. Lam, and D. Schübeler. 2005. Chromosome-wide and promoter-specific analyses identify sites of differential DNA methylation in normal and transformed human cells. Nature Genet 37:853-862.

25.Weber, M., I. Hellmann, M.B. Stadler, L. Ramos, S. Pääbo, M. Rebhan, and D. Schübeler. 2007. Distribution, silencing potential and evolutionary impact of promoter DNA methylation in the human genome. Nature Genet. 39:457-466.

26. Keshet, I., Y. Schlesinger, S. Farkash, E. Rand, M. Hecht, E. Segal, E. Pikarski, R.A. Young, A. Niveleau, H. Cedar, and I. Simon. 2006. Evidence for an instructive mechanism of de novo methylation in cancer cells. Nature Genet. 38:149-153.

27. Jacinto, F.V., E. Ballestar, S. Ropero, and M. Esteller. Discovery of epigenetically silenced genes by methylated DNA immunoprecipitation in colon cancer cells. Cancer Res. (In press.)

28.Barski, A., S. Cuddapah, K. Cui, T.Y. Roh, D.E. Schones, Z. Wang, G. Wei, I. Chepelev, and K. Zhao. 2007. High-resolution profiling of histone methylations in the human genome. Cell 129:823-837.

29. Bibikova, M., Z. Lin, L. Zhou, E. Chudin, E.W. Garcia, B. Wu, D. Doucet, N.J. Thomas, Y. Wang, E. Vollmer, et al. 2006. Highthroughput DNA methylation profiling using universal bead arrays. Genome Res. 16:383-393. 